\title{
Minigaps and Novel Giant Negative Magnetoresistance in InAs/GaSb Semimetallic Superlattices
}

\author{
M. Lakrimi,${ }^{1}$ S. Khym, ${ }^{1}$ R. J. Nicholas, ${ }^{1}$ D. M. Symons,${ }^{2}$ F. M. Peeters,${ }^{3}$ N. J. Mason, ${ }^{1}$ and P. J. Walker ${ }^{1}$ \\ ${ }^{1}$ Department of Physics, Clarendon Laboratory, University of Oxford, Parks Road, Oxford OX1 3PU, United Kingdom \\ ${ }^{2}$ Grenoble High Magnetic Field Laboratory, MPI-FKF and CNRS, BP 166, F-38042 Grenoble CEDEX 9, France \\ ${ }^{3}$ Department of Physics, University of Antwerp (UIA), Universiteitsplein 1, B-2610 Antwerpen, Belgium
}

(Received 4 February 1997)

\begin{abstract}
The magnetoresistance of semimetallic InAs/GaSb superlattices has been measured in parallel magnetic fields. The temperature dependence reveals for the first time the existence of a minigap at the anticrossing points between the electron and hole dispersion relations. At high magnetic fields, samples which are close to intrinsic show surprisingly large decreases in resistance of up to 70\%. The magnetoresistance also depends on crystal orientation. We present a theoretical model which explains the experimental observations and estimates the minigap to be of order $7 \mathrm{meV}$. [S0031-9007(97)04291-9]
\end{abstract}

PACS numbers: 72.20.My, 68.65.+g, 72.80.Ey

There has been a long-standing controversy over whether the broken gap system InAs/GaSb [1] should behave as a semiconductor or a semimetal. Long period superlattices exhibit electron transfer from the valence band of the GaSb layers into the conduction band of the InAs, leading at first sight to semimetallic behavior. Considering the in-plane carrier motion, however, the dispersion relations of the electrons and holes cross, and due to mixing of the bands a small minigap should form, leading to the reestablishment of semiconducting behavior, as was first predicted by Altarelli [2]. In practice experimental evidence for this minigap has remained very scarce. Measurements such as magnetotransport have been interpreted within the semimetallic picture with the simultaneous presence of electrons and holes [3], particularly for extrinsic structures in which the electron and hole densities are not equal.

In this Letter, we present the first results in which there is clear evidence for the presence of this minigap which causes a strong temperature dependent resistivity. We show how this gap is removed by applying a parallel magnetic field which shifts the position of the conduction and valence bands relative to each other in $k$ space. This causes a transition to true semimetallic character with an indirect band overlap, and as a result there is a giant negative magnetoresistance in structures which have carrier concentrations determined primarily by intrinsic charge transfer. We also present a calculation which shows that the minigap which is formed is dependent on the direction of the $k$ vector, leading to some indirect overlap of the bands despite the existence of local energy gaps. This resolves the dilemma of why many structures which were, in principle, expected to be semiconducting still show primarily metallic conduction at low temperatures.

A series of InAs/GaSb superlattices were grown by metal-organic vapor phase epitaxy consisting of either 20, 40 , or 80 layers of InAs bounded by as many layers of
GaSb [4]. Some studies were also made on double heterojunctions (DHETs) consisting of a single layer of InAs of thickness typically $300 \AA$ sandwiched between thick $\mathrm{GaSb}$ layers. Our previous studies of the magnetotransport of thick layer structures revealed that the main source of carriers is the intrinsic charge transfer from the GaSb layers to the InAs layers [5]. Estimates of the electron and hole mobilities from fits using conventional two carrier Hall effect formulas indicate, for samples with InAs layer thicknesses in excess of $200 \AA$, values of order 80000 and $10000 \mathrm{~cm}^{2} / \mathrm{V} \mathrm{s}$, respectively, with electron to hole density ratios in the range 2 to 1.05 . However, the present measurements indicate that these values are probably severely limited by the presence of the minigap induced by electron-hole mixing rather than the quality of the material. Resistivity measurements were made as a function of temperature and magnetic field using conventional Hall bars, with the current direction oriented along both the [100] and the [110] directions, and the magnetic field both perpendicular and parallel to the current.

Figure 1 shows the electrical resistance as a function of magnetic field for a 40 period [120 ̊ (InAs) and $100 \AA$ $(\mathrm{GaSb})]$ superlattice (SL) for temperatures ranging from 80 to $4.2 \mathrm{~K}$, with the magnetic field applied parallel to the SL planes and perpendicular to the current direction. It needs to be emphasized that, for such narrow layers, eight-band self-consistent $\mathbf{k} \cdot \mathbf{p}$ calculations [6] clearly demonstrate that this SL has only one subband populated. The high field resistivity is almost temperature independent, while by contrast the zero field resistance increases rapidly with falling temperature as for a bulk intrinsic semiconductor. By $4.2 \mathrm{~K}$ a giant negative magnetoresistance has appeared, corresponding to a fourfold decrease in resistance by $12 \mathrm{~T}$. The low perpendicular field Hall coefficient at $4.2 \mathrm{~K}$ suggests an electron density of about $2 \times 10^{11} \mathrm{~cm}^{-2}$ for this sample. However, when two carrier fits are made to the low perpendicular 


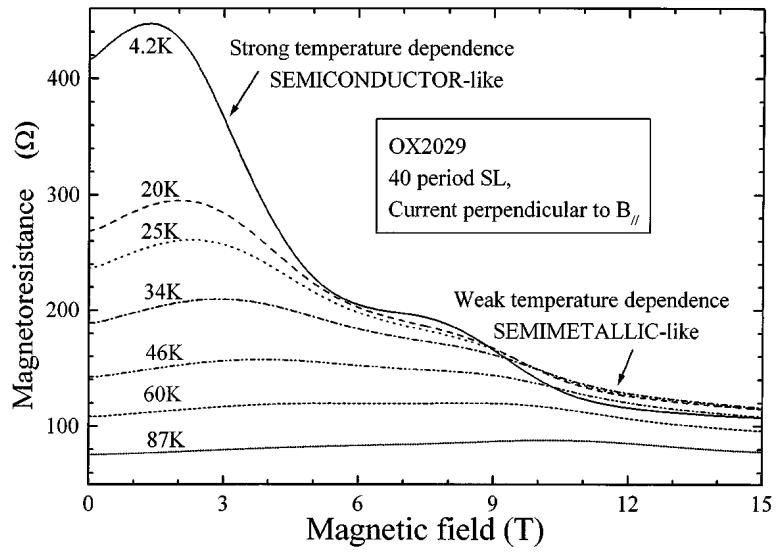

FIG. 1. Temperature dependence of the magnetoresistance for a $120 \AA$ (InAs)/100 $\mathrm{A}(\mathrm{GaSb}) 40$ period superlattice with magnetic field applied parallel to the superlattice planes.

field Hall coefficient in the presence of an additional constant parallel field component of $11 \mathrm{~T}$, the electrons and holes give classical two carrier behavior with electron (hole) densities and mobilities of $4.7(1.8) \times 10^{11} \mathrm{~cm}^{-2}$ and $11000(5200) \mathrm{cm}^{2} / \mathrm{V} \mathrm{s}$, respectively. This change is due to the transition to true two carrier semimetallic behavior brought about by the parallel field component.

The origin of the temperature dependence is directly related to the small minigap caused by the mixing of the electron and hole dispersion relations as shown at zero field in Fig. 2(a). This causes a strong reduction of the group velocity of the charge carriers close to the Fermi energy, thus increasing the resistivity at low temperatures. In Figs. 2(b) and 2(c), the parallel field has induced a relative shift of the electron and hole dispersion relations proportional to their relative displacement in real space, $\Delta z$. As the field is increased the regions where there is

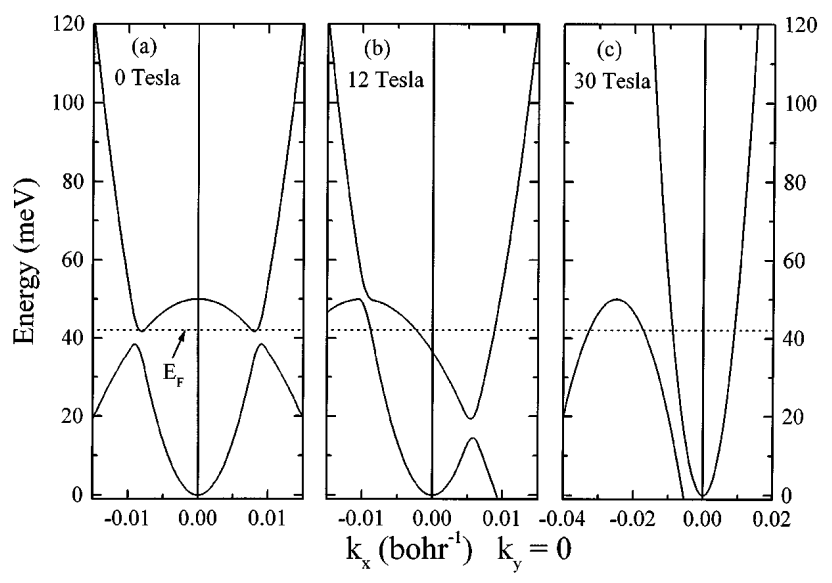

FIG. 2. Diagram showing how the electron and hole dispersion relations move away from each other with parallel magnetic field. The SL period and the separation between the electron and hole confinement energies are $200 \AA$ and $50 \mathrm{meV}$, respectively. The $x$ axis has been changed in (c) for clarity. $k_{x}$ is taken perpendicular to the SL axis. strong band mixing and therefore reduced group velocity move away from the Fermi energy and the resistivity falls. In the high field limit the electron-hole mixing is removed and a fully semimetallic band structure is produced, as shown in Fig. 2(c).

In order to model the resistivity we have used the relaxation time approximation in Boltzmann transport theory to calculate the contribution to the conductivity from all states close to the Fermi level. A similar approach has been used to model the behavior of structures with two parallel 2D electron gases [7]. We have specifically taken into account the modified dispersion relations caused by the interband coupling and the anisotropy of the valence band. To simplify the calculations, we have used a two band model which includes an in-plane magnetic field to give the carrier dispersion relations shown in Fig. 2 and have assumed the $2 \mathrm{D}$ limit of no dispersion in the superlattice growth direction. The energies are given by

$$
\begin{aligned}
E_{ \pm}(\vec{k})=\frac{1}{2}\left\{\left[E_{e}^{0}(\vec{k})+E_{h}^{0}(\vec{k}-\delta \vec{k})\right]\right. & \\
& \left. \pm \sqrt{\left[E_{e}^{0}(\vec{k})-E_{h}^{0}(\vec{k}-\delta \vec{k})\right]^{2}+\Delta^{2}}\right\},
\end{aligned}
$$

where $E_{e}^{0}(\vec{k})$ and $E_{h}^{0}(\vec{k})$ are the electron and hole energies of the uncoupled system. $\Delta$ is the minigap or coupling energy and is taken as constant. The isotropic electron $\left(m_{e}=0.03 m_{0}\right)$ band and the warped hole band are given by $E_{e}^{0}(\vec{k})=\hbar^{2} \vec{k}^{2} / 2 m_{e}$ and $E_{h}^{0}(\vec{k})=E g-$ $\hbar^{2} / m_{0}\left[|A| \vec{k}^{2}-\left(B^{2} \vec{k}^{4}+C^{2} k_{x}^{2} k_{y}^{2}\right)^{1 / 2}\right]$, respectively. $E_{g}$ is the energy overlap separating the electron and hole confinement energies. The constants $A, B$, and $C$ are taken as $-14.3,-10.4$, and 13.5 , respectively [8]. Adding an inplane magnetic field has several effects. The main effect, which is dominant at low fields, is a shift of the centers of the electron and hole dispersions in $k$ space through $\delta k_{x}$ and $\delta k_{y}$. For a magnetic field applied in the [010] direction $\delta k_{x}=e B_{\|} \Delta z / \hbar, \delta k_{y}=0$, and in the [110] direction $\delta k_{x}=-\delta k_{y}=e B_{\|} \Delta z / 2^{1 / 2} \hbar$. We have neglected any diamagnetic energy shifts of the confinement energies or reconstruction of the states to form Landau levels. Intersubband measurements performed using a magnetic field parallel to the layers have shown that for thin superlattices (InAs of order $140 \AA$ ) the subband energies exhibit little dependence on parallel magnetic field [9]. These studies also show that it is only for InAs thicknesses larger than $400 \AA$ that parallel fields of order $10 \mathrm{~T}$ are able to destroy the zero field band structure and produce Landau levels [9]. The conductivity is calculated using

$$
\sigma_{x x}=\frac{2 e^{2}}{A} \sum_{i, \vec{k}} v_{x}^{2}(i, \vec{k}) \tau_{0}\left[-\frac{\partial f[E(i, \vec{k})]}{\partial E(i, \vec{k})}\right] .
$$

where $f[E(i, k)]$ is the Fermi distribution, $\boldsymbol{v}_{x}$ a component of the group velocity, $\tau_{0}$ the scattering time, and $A$ is a normalization area. The sum is over all $k$ states and bands $(i)$, and since the band structure is highly anisotropic, it is 
imperative to consider all directions of the $k$ vector. Since $\rho_{x y}=0$, then $\rho_{x x}=1 / \sigma_{x x}$.

Figure 3 shows the results of simulations of the resistivity for a structure in which the energy separation between the electron and hole confinement energies is fixed at $40 \mathrm{meV}$. This value was chosen to give carrier densities close to the numbers deduced from the two carrier fits to the Hall resistivity in the presence of a large parallel field component. To illustrate the sensitivity of the calculation to given parameters, a range of minigaps $(\Delta)$ and Fermi energies $\left(E_{F}\right)$ are chosen. The main effect of varying the Fermi energy is to change the electronto-hole density ratio in the structure. The best fitted $\Delta z$ is $190 \AA$ which is close to the SL period. Figure 3(a) shows the effect of increasing the size of $\Delta$ for fixed electron and hole densities (fixed $E_{F}$ ). The magnitude of the resistance change increases with $\Delta$, and also the position of the features changes. The behavior is even more sensitive to $E_{F}$, as can be seen in Fig. 3(b). When $E_{F}$ lies in the minigap the electron and hole densities become comparable, and the resistivity depends critically on both the size of the gap and the band parameters of the materials. Overall, based on the positions of the features in field and the shape of the curve, we consider curve 2 with a coupling value of $7 \mathrm{meV}$ to be the best fit to the data. Furthermore, we have also modeled the temperature dependence of the zero field magnetoresistance and found that a minigap of $7 \mathrm{meV}$ yields the best fit to the data. This value of $\Delta=7 \mathrm{meV}$ is similar to the optically estimated anticrossing between Landau levels from different subbands on slightly thicker

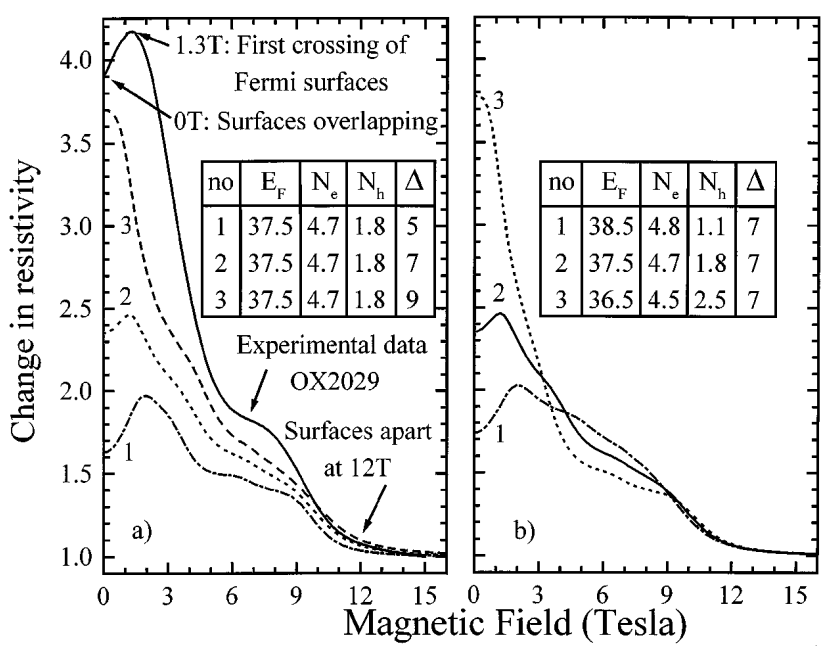

FIG. 3. Experimental data from Fig. 1 and theoretical simulations performed at $4.2 \mathrm{~K}$ using various (a) coupling values and (b) Fermi energies $\left(E_{F}\right)$ measured relative to the bottom of the electron subband. $\Delta z$ and the separation between the electron and hole confinement energies were fixed to $190 \AA$ and $40 \mathrm{meV}$, respectively. The data was ratioed against the value at $15 \mathrm{~T}$. The electron $\left(N_{e}\right)$ and hole $\left(N_{h}\right)$ densities are given in units of $10^{11} \mathrm{~cm}^{-2} . E_{F}$ and $\Delta$ are in $\mathrm{meV}$. structures [10] and that calculated from conductionvalence Landau level mixing effects [11]. It also matches the value found from self-consistent eight-band $\mathbf{k} \cdot \mathbf{p}$ calculations for zero in-plane magnetic field.

Figure 3 shows that the overall simulation corresponds well with the experimental data, including the presence of two peak features at about 2 and $8 \mathrm{~T}$. Qualitatively, this behavior is related to the shift of the Fermi surfaces of the electrons and holes. Figure 4 shows the evolution of the Fermi surface for four values of parallel field. At low field the Fermi surface is strongly distorted due to the band coupling. If we ignore the band coupling, we see that the two peaks arise when the electron and hole Fermi surfaces cross, corresponding approximately to the condition

$$
\Delta k=\frac{e B_{\|} \Delta z}{\hbar}=k_{F}^{e}+k_{F}^{h},
$$

where $k_{F}^{e}$ and $k_{F}^{e}$ are the electron and hole Fermi wave vectors. As $B_{\|}$is increased the two Fermi surfaces experience a first crossing at about $1.8 \mathrm{~T}$ and then become independent at $9 \mathrm{~T}$, leading to semimetallic behavior with no anticrossing gap and a weak temperature dependence of the resistance. The formation of the full $k$-space indirect semimetallic band structure is shown schematically in Fig. 2 as a function of $B_{\|}$. Using a range of modeling parameters, we estimate that the minigap is of order $7 \mathrm{meV}$ in samples with layer thicknesses in the range $100-300 \AA$. With these values the valence band anisotropy is sufficiently large that for the majority of structures some residual semimetallic character still remains even at $B=0 \mathrm{~T}$. Consequently, the increase in resistivity saturates below $4 \mathrm{~K}$.

The negative magnetoresistance is a general feature of all of the superlattices studied and becomes gradually less

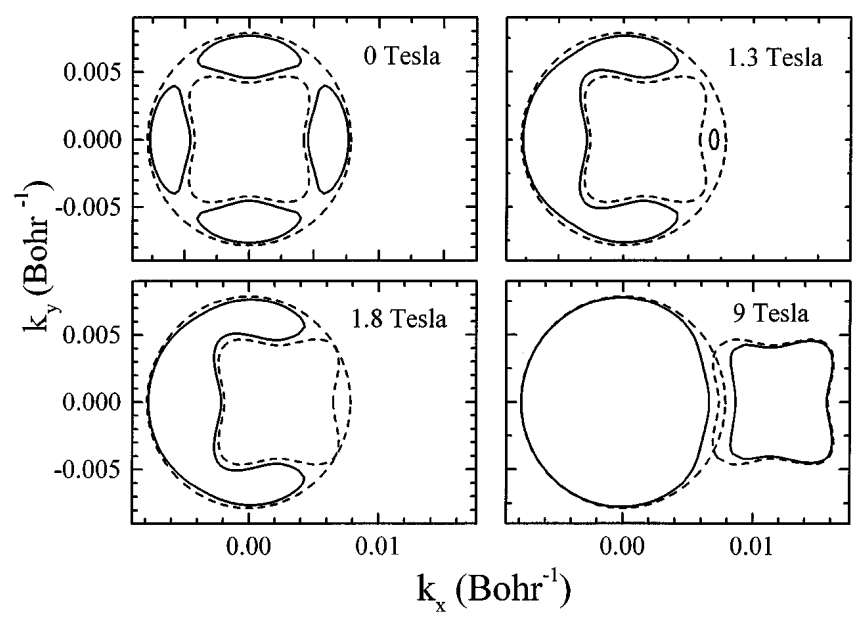

FIG. 4. Contour maps of the Fermi surface at various values of parallel magnetic field. The solid line is calculated with the mixing included and the dotted line with completely independent electrons and holes. 
pronounced as the layer thicknesses increase, probably because the anisotropy of the hole dispersion leads to a larger indirect overlap of the bands when the Fermi wave vector and, hence, the carrier densities are larger. Similar features have also been observed in DHETs, provided that the majority of the carriers present come from intrinsic charge transfer. A few DHETs were also studied in which only electrons were present, due to the use of a very thin capping layer at the surface. The negative magnetoresistance was then absent [12].

In addition, we would expect that the anisotropic nature of the valence band in III-V semiconductors should lead to a crystal orientation dependence of the negative magnetoresistance. This is illustrated in Fig. 5, which shows the resistivity and its first derivative from a set of longer period superlattice structures fabricated from the same wafer with the magnetic field oriented along the [010], [100], [110], and [110] directions and the current always perpendicular to magnetic field. The first derivative of the resistance shows a clear symmetry with crystal orientation, with a number of additional features coming from the structures oriented along [010] and [100]. This is due to the different amounts of electron-hole hybridization which occur when the valence bands are shifted in different directions in $k$ space. More detailed modeling may enable the quantitative mapping of the complicated valence band dispersion relations as seen in $B$-parallel shifted tunneling measurements [13].

One important factor which has been neglected in our simplified approach is any change in the magnitude of the scattering time $\tau_{0}$. It is well known that relatively weak negative magnetoresistance effects are seen in multiple subband single carrier systems in parallel magnetic field,

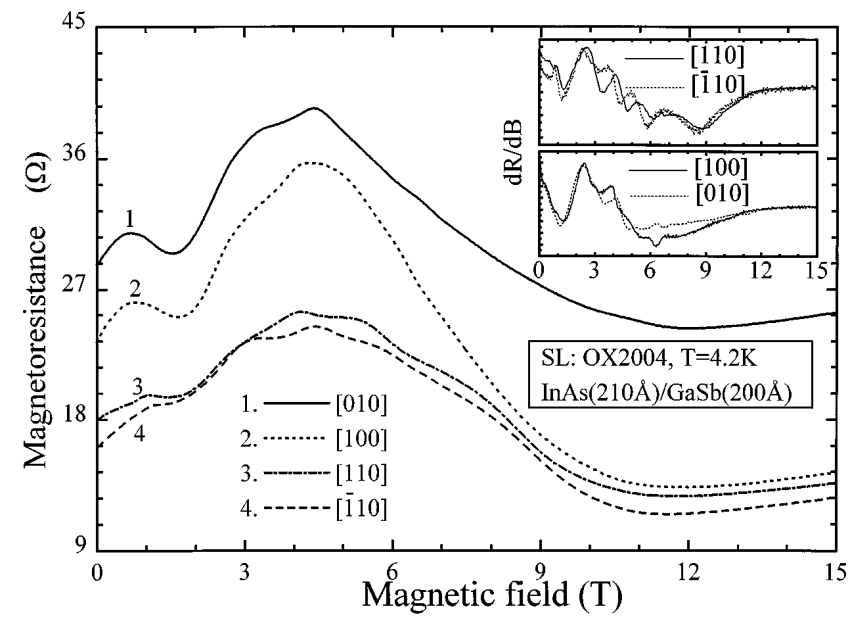

FIG. 5. Magnetoresistance in parallel magnetic field for four different Hall bars made from the same wafer but oriented along different directions. The two insets show the first derivative of the data, highlighting the pairing in the symmetry between the four samples. OX2004 is a 20 period SL with $4.2 \mathrm{~K}$ electron (hole) density of $8.9(7.9) \times 10^{11} \mathrm{~cm}^{-2}$ and mobility of $106000(7400) \mathrm{cm}^{2} / \mathrm{V} \mathrm{s}$. because of the suppression of intersubband scattering due to subband depopulation effects [14]. The inclusion of changes in intersubband or interband scattering contributions as the levels move through each other would lead to extra features and an even larger negative magnetoresistance. This would improve the quantitative agreement between experiment and theory which at present gives too small a value for the negative magnetoresistance (Fig. 3). Unfortunately, such an approach would require several additional input parameters, and our purpose in this Letter is to point out that the primary origin of the effects reported here is the presence and control of the additional small energy gap caused by interband coupling.

In conclusion, therefore, we can say that we have produced the first conclusive proof of the existence of an energy gap of about $7 \mathrm{meV}$ which is induced by band mixing. Furthermore, we have presented a simple theoretical model which allows us to model the data. The magnetoresistance features were found to depend on crystal orientation because of the anisotropic nature of the valence band.

This work is funded by EPSRC (UK). F.M.P. and D. M. S. acknowledge the support of the HCM and TMR programs of the European Union, respectively.

Note added. - Since submitting this Letter, Yang et al. [Phys. Rev. Lett. 78, 4613 (1997)] have also shown evidence for the existence of the minigap in this system.

[1] L. L. Chang et al., Appl. Phys. Lett. 35, 939 (1979).

[2] M. Altarelli, Phys. Rev. B 28, 842 (1983).

[3] E.E. Mendez et al., Phys. Rev. Lett. 55, 2216 (1985); M. S. Daly et al., Phys. Rev. B 53, R10 524 (1996).

[4] G. R. Booker et al., J. Cryst. Growth 145, 778 (1994); ibid. 146, 495 (1995).

[5] M. S. Daly et al., Surf. Sci. 361-362, 205 (1996).

[6] T. A. Vaughan, D.Phil. thesis, University of Oxford, 1996 (unpublished), Chap. 7.

[7] J. A. Simmons et al., Phys. Rev. Lett. 73, 2256 (1994); T. Ihn et al., Phys. Rev. B 54, 2315 (1996).

[8] Landolt-Börnstein Numerical Data and Functional Relationships in Science and Technology, New Series Group III (Springer-Verlag, Berlin, 1982), Vol. 17, pp. 19-20.

[9] A. J. L. Poulter et al., Physica B (to be published).

[10] M. Lakrimi et al., in Proceedings of the 23rd International Conference on the Physics of Semiconductors, edited by M. Scheffler and R. Zimmermann (World Scientific, Singapore, 1996), Vol. 3, p. 1707.

[11] J.-C. Chiang et al., Phys. Rev. Lett. 77, 2053 (1996).

[12] D. M. Symons et al., Ref. [10], p. 2207.

[13] R. K. Hayden et al., Phys. Rev. Lett. 66, 1749 (1991); L. Eaves et al., Surf. Sci. 263, 199 (1992).

[14] Z. Schlesinger et al., Phys. Rev. Lett. 50, 2098 (1983); D. R. Leadley et al., Semicond. Sci. Technol. 5, 1081 (1990). 